\title{
OPEN Machine learning-based prediction model using clinico-pathologic factors for papillary thyroid carcinoma recurrence
}

\author{
Young Min Park ${ }^{1 \bowtie}$ \& Byung-Joo Lee Le $^{2 \bowtie}$
}

This study analyzed the prognostic significance of clinico-pathologic factors, including the number of metastatic lymph nodes (LNs) and lymph node ratio (LNR), in patients with papillary thyroid carcinoma (PTC), and attempted to construct a disease recurrence prediction model using machine learning techniques. We retrospectively analyzed clinico-pathologic data from 1040 patients diagnosed with PTC between 2003 and 2009. We analyzed clinico-pathologic factors related to recurrence through logistic regression analysis. Among the factors that we included, only sex and tumor size were significantly correlated with disease recurrence. Parameters such as age, sex, tumor size, tumor multiplicity, ETE, ENE, $\mathrm{pT}$, pN, ipsilateral central LN metastasis, contralateral central LNs metastasis, number of metastatic LNs, and LNR were input for construction of a machine learning prediction model. The performance of five machine learning models related to recurrence prediction was compared based on accuracy. The Decision Tree model showed the best accuracy at $95 \%$, and the lightGBM and stacking model together showed $93 \%$ accuracy. Among those factors mentioned above, LNR and contralateral LN metastasis were used as important features in all machine learning prediction models. We confirmed that all machine learning prediction models showed an accuracy of $90 \%$ or more for predicting disease recurrence in PTC. LNR and contralateral LN metastasis were used as important features for constructing a robust machine learning prediction model. In the future, we have a plan to perform large-scale multicenter clinical studies to improve the performance of our prediction models and verify their clinical effectiveness.

Over the past 20 years, the incidence of thyroid cancer has increased rapidly, and the most common histologic type of thyroid cancer is papillary thyroid carcinoma (PTC) ${ }^{1}$. PTC has an excellent prognosis and a better survival rate than other carcinomas; however, disease ultimately recurs in about 5-21\% of PTC patients ${ }^{2,3}$. In PTC patients with recurrent disease, surgical treatment is generally required, and re-operation poses additional medical costs and significant morbidity, compared to the initial surgery. Therefore, preventing recurrence in PTC patients can reduce and deteriorations in quality of life related to reoperation.

According to previous reports, tumor size, extrathyroidal extension (ETE), age, lymph node (LN) metastasis, tumor multiplicity, and extranodal spread (ENE) are known risk factors for recurrence of PTC ${ }^{4-6}$. In PTC, LN metastasis occurs in 20-90\% of patients, and the number of metastatic LNs and lymph node ratio (LNR), representing metastatic LN burden, are known to be important prognostic factors associated with recurrence of $\mathrm{PTC}^{7-13}$. Since various clinico-pathological factors and nodal factors (i.e., number of metastatic LNs and LNR) have been shown to be related to the recurrence of PTC, these factors should be considered in an integrated manner to establish a disease recurrence prediction model.

The 8th TNM staging system was revised by the American Joint Committee on Cancer (AJCC) to more accurately predict the disease-specific survival of PTC patients. However, it does not reflect the biological behavior of PTC and has limitations in predicting the risk of recurrence ${ }^{14-16}$. In particular, the number and size of metastatic LNs are not reflected in the revised TNM staging system. Also, the N classification of the revised TNM stage

\footnotetext{
${ }^{1}$ Department of Otorhinolaryngology, Gangnam Severance Hospital, Yonsei University College of Medicine, 211 Eonju-ro, Gangnam-gu, Seoul 06273, Korea. ${ }^{2}$ Department of Otorhinolaryngology-Head and Neck Surgery, Pusan National University School of Medicine, Pusan National University and Biomedical Research Institute, Pusan National University Hospital, 1-10 Ami-Dong, Seo-Gu, Busan 602-739, Korea. ${ }^{\square}$ email: autumnfe79@yuhs.ac; voiceleebj@gmail.com
} 
system merely comprises three groups and does not consider other nodal factors ${ }^{17-22}$. We presume that the more prognostic factors, such as number of metastatic LNs and LNR, that can be integrated into the TNM system, the more accurate the prediction model could be for predicting disease recurrence of PTC patients.

Machine learning technology is widely used in the medical field, especially in the fields of radiology, ophthalmology, and dermatology ${ }^{23-28}$. However, studies on the construction of machine learning models that can be used to predict disease recurrence of PTC are extremely rare. Establishing a robust predictive model of PTC could help with selecting high-risk patients for intensified treatment tailored according to risk stratification and with suggesting candidates for active follow-up. This study analyzed the prognostic significance of various clinico-pathologic nodal factors, including the number of metastatic LNs and LNR, in patients with PTC patients and attempted to construct a disease recurrence prediction model based on these factors using machine learning techniques.

\section{Materials and methods}

This study was approved by the Institutional Review Board (IRB) of Pusan University. Informed consent was not obtained from any participants because the IRB waived the need for individual informed consent. This retrospective research was performed in accordance with the Declaration of Helsinki. Medical data of patients diagnosed and treated for PTC at Pusan National University Hospital from June 2003 to December 2009 were analyzed retrospectively. We included patients who were diagnosed with PTC and underwent total thyroidectomy and central neck dissection with/without lateral neck dissection. We excluded (1) cases with a distant metastasis at the time of diagnosis, (2) patients who received previous surgery or radiotherapy to the head and neck area, and (3) cases with insufficient clinical data that were lost to follow-up after surgery. Finally, 1040 patients were included in the study, including 147 males and 893 females. Their ages ranged from 13 to 79 years and the mean age was 48.5 years. Tumor stage was classified according to the 8th AJCC staging system.

To detect disease recurrence, all patients underwent physical examination, ultrasound, and thyroglobulin measurement every 6-12 months after surgery. If necessary, additional imaging studies such as computed tomography, whole body iodine scan, and positron emission tomography were performed. Recurrence was defined as a case in which a new lesion was detected that was not previously observed in the imaging studies and which was pathologically confirmed through fine needle aspiration cytology.

Tumor size, ETE, multiplicity, ENE, and TNM stage were analyzed. The surgical specimens from central neck dissection were divided into ipsilateral and contralateral areas according to the location of the tumor, and therein the number of metastatic LNs and the total number of harvested LNs were recorded. LNR was calculated by dividing the number of metastatic LNs by the total number of harvested LNs. The cut-off value of LNR was determined in consideration of optimized sensitivity and specificity for predicting disease recurrence using receiver operating characteristic curves (ROC). The optimal value of LNR was used to construct machine learning models for predicting disease recurrences.

Machine learning was performed based on the supervised learning method, and a variety of machine learning models was used including the decision tree, random forest, XGBoost, and LightGBM, and Stacking models. Learning was performed with the five models mentioned above, and accuracy was used to evaluate model performance. Scikit-learn version 12.3 was used for model building and learning. $80 \%$ of the data set was classified as the training set and was used for learning, and the remaining $20 \%$ was used as a test set. To account for selection bias, the five-fold-cross-validation technique was applied.

Patient clinical information, pathologic information, recurrence, and cause of recurrence were analyzed. The Chi-square or independent two-sample t-test were used to evaluate differences in variables between two independent groups. The multivariate Cox proportional hazards regression model was used to evaluate the effect of several variables on disease recurrence. $p$ values $<0.05$ were considered indicative of statistical significance. Statistical analyses were performed using python 3.8 version and SPSS version 25.0 for Windows (SPSS, Chicago, IL).

\section{Results}

A total of 1040 patients were included in this study, and all patients underwent total thyroidectomy and central neck dissection. In total, 180 patients $(17.3 \%)$ underwent lateral neck dissection simultaneously due to lateral LN metastasis. The average tumor size was $12.4 \mathrm{~mm}$ (range 2-125). ETE findings were observed in 586 (56.3\%) patients, and tumor multiplicity was observed in 178 (17.1\%) patients. ENE findings were observed in 159 patients (15.3\%). With respect to T classification, 508 patients were classified as T1, 46 patients were T2, 483 patients were $\mathrm{T} 3$, and three patients were T4. The average number of metastatic LNs in the central compartment was 1.73 (range $0-19$ ), and the average number of LNs removed was 8.32 (range $0-36$ ). The average LNR value was 0.20 (range $0-1$ ). The mean follow-up period was 79.0 months (range 46-149), and the total number of recurrence events during the study period was 41 . Other clinico-pathological information is summarized in Table 1.

The cut-off value for the LNR was set to show the optimal sensitivity and specificity for recurrence prediction. Regarding the prediction of recurrence, LNR showed a statistically significant correlation ( $p$ value $=<0.001$ ), with an AUC value of 0.752 , and an LNR value of 0.24 was set as the optimal cut-off value. There were 519 patients (49.9\%) with an LNR-of 0; 179 (17.2\%) with an LNR value greater than 0, but less than 0.24; and 342 (32.9\%) with an LNR value of 0.24 or more. Recurrence-free survival was significantly lower in the patient group with LNR $>0.24$, compared to the other two groups (Fig. 1A). A cut-off value for the number of metastatic LNs was devised to obtain optimal sensitivity and specificity for recurrence prediction. The number of metastatic LNs was statistically significantly correlated with recurrence ( $p$ value $=<0.001$ ), with an AUC value of 0.742 . A value of 2 was set as the cut-off for the number of metastatic LNs. There were 519 patients (49.9\%) with 0 metastatic LNs, 161 (15.5\%) with one LN metastasis, and 360 (34.6 with two or more LN metastases. Recurrence-free survival 


\begin{tabular}{|c|c|}
\hline Variable & No. of patients (\%) \\
\hline Mean age, $y$ (range) & $48.5(13-79)$ \\
\hline \multicolumn{2}{|l|}{ Sex } \\
\hline Male & $147(14.1)$ \\
\hline Female & $893(85.9)$ \\
\hline \multicolumn{2}{|c|}{ Extrathyroidal extension } \\
\hline Yes & $586(56.3)$ \\
\hline No & $454(46.7)$ \\
\hline \multicolumn{2}{|l|}{ Tumor multiplicity } \\
\hline Yes & $178(17.1)$ \\
\hline No & $862(82.9)$ \\
\hline \multicolumn{2}{|c|}{ Extranodal extension } \\
\hline Yes & $159(15.3)$ \\
\hline No & $881(84.7)$ \\
\hline \multicolumn{2}{|l|}{ pT classification } \\
\hline 1 & $508(48.8)$ \\
\hline 2 & $46(4.4)$ \\
\hline 3 & $483(46.4)$ \\
\hline 4 & $3(0.2)$ \\
\hline \multicolumn{2}{|l|}{ pN classification } \\
\hline N0 & $506(48.7)$ \\
\hline N1a & $354(34.0)$ \\
\hline N1b & $180(17.3)$ \\
\hline
\end{tabular}

Table 1. Clinical information for all patients $(n=1040)$ enrolled in the study.

was analyzed by dividing patients into these three groups and was significantly decreased lower in patients with two or more metastatic LNs (Fig. 1B).

We analyzed the association between clinico-pathologic factors and recurrence through univariate analysis. Sex, tumor size, ETE, pT classification, pN classification, number of metastatic LNs, and LNR were significantly correlated with disease recurrence. Clinico-pathologic factors related to recurrence were also analyzed with logistic regression for multivariate analysis. Among the factors included in the analysis, only sex and tumor size showed significant correlations with disease recurrence (Table 2).

To build a machine learning prediction model, the algorithm was trained using parameters of age, sex, tumor size, tumor multiplicity, ETE, ENE, pT, pN, ipsilateral central LN metastasis, contralateral central LN metastasis, number of metastatic LNs, and LNR. Since disease recurred in only 41 of 1040 cases, the SMOTE technique was applied to adjust for the imbalance in learning data. The performance of five machine learning models for recurrence prediction was compared based on accuracy. The decision tree model showed the best accuracy at $95 \%$, and the LightGBM and stacking models showed accuracies of 93\%. Table 3 summarizes the performance of the five models. The tree structure of the decision tree model was visualized using graphic software, and feature importance was also visualized and analyzed (Fig. 2). In other machine learning models, feature importance was explored to determine the major factors that influence the prediction of recurrence in PTC patients. Although the feature importance results differed slightly between machine learning models, LNR and contralateral LN metastasis were consistently important features in all models (Table 4).

\section{Discussion}

The revised 8th TNM staging system is suitable for assessing the risk of death in patients with PTC, but not for predicting the risk of recurrence. Age, aggressive histology, tumor size, and LNs metastasis are known risk factors associated with PTC recurrence ${ }^{29}$. The 2015 American Thyroid Association guidelines suggested the number and size of metastatic LNs and ENE as risk factors for recurrence ${ }^{30,31}$. Meanwhile, LNR, calculated by dividing the number of metastatic LNs by the total number of removed LNs, has been reported in previous studies as a risk factor for recurrence of $\mathrm{PTC}^{13,32,33}$. Lee et al. ${ }^{34}$ reported that the performance of recurrence prediction increased when LNR was incorporated into the existing 2015 ATA risk stratification. In our study, the sensitivity and specificity for predicting disease recurrence were optimized when an LNR value of 0.24 was set as a cut-off. The number of metastatic LNs also showed a statistically significant correlation with the prediction of disease recurrence when 2 or more LNs was set as the cut-off value. To construct a robust prediction model for disease recurrence in PTC, these all factors mentioned above should be considered and integrated into the model.

In univariate analysis of risk factors for PTC recurrence, sex, tumor size, ETE, pT, pN, number of metastatic LNs, and LNR were significantly correlated with recurrence. In multivariate analysis using logistic regression, only tumor size showed a significant correlation with disease recurrence. Because logistic regression was used to analyze prognostic factors based on a linear combination between variables, if the degree of correlation between variables was high, the analysis was limited with overfitted results. On the other hand, since machine learning 


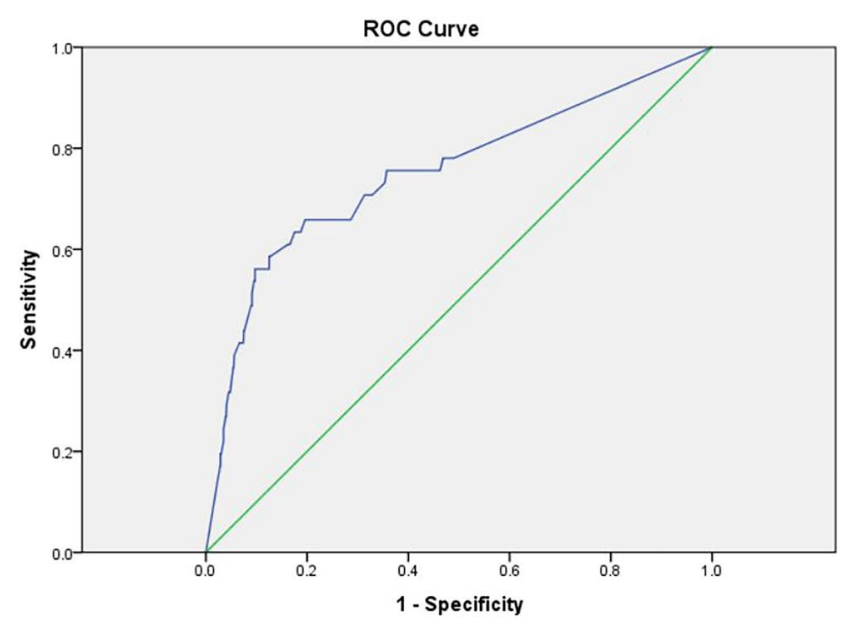

A

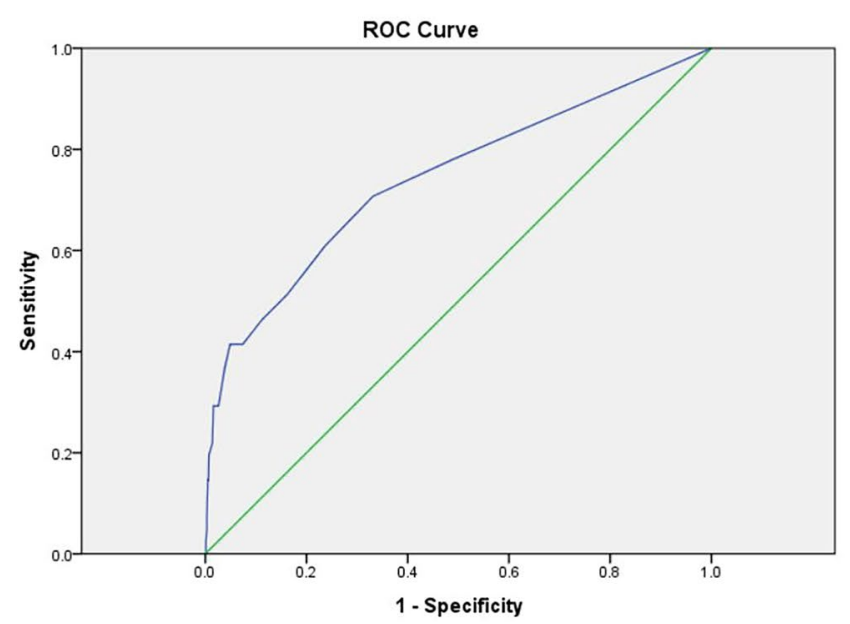

A

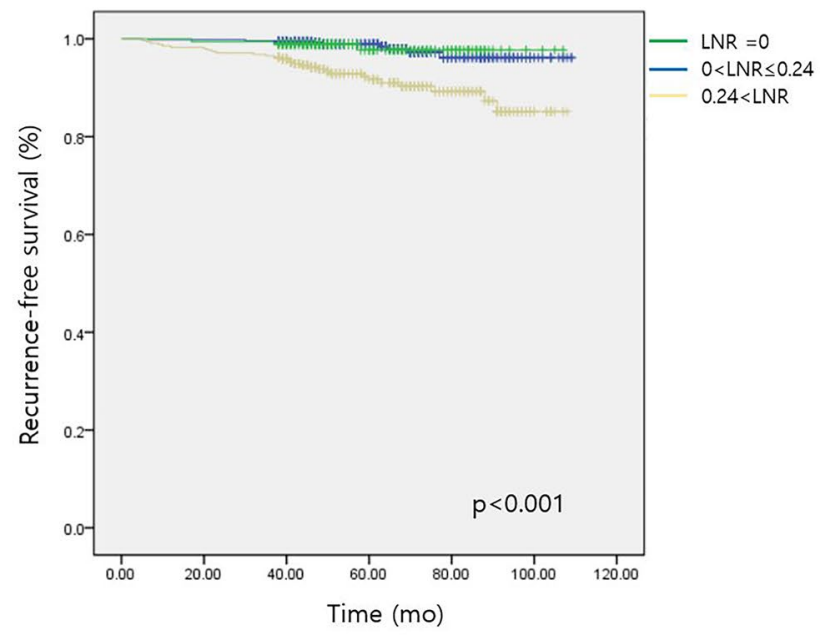

B

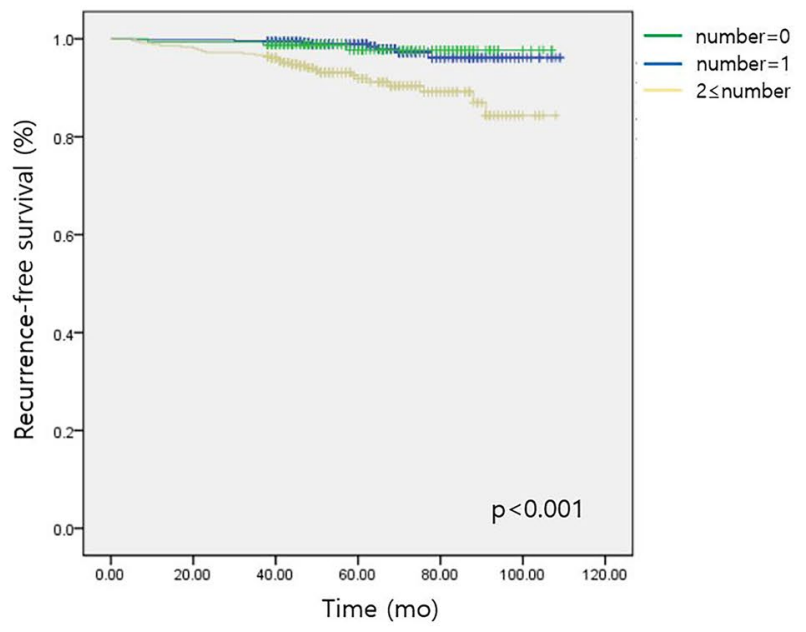

B

Figure 1. (A) The cut-off value for the lymph node ratio (LNR) was determined in consideration of the sensitivity and specificity optimized to predict disease recurrence using a receiver operating characteristic curve (ROC). Recurrence-free survival of the patient group with LNR $>0.24$ was significantly decreased. (B) The cut-off value for the number of metastatic LNs was determined in consideration of the sensitivity and specificity optimized to predict disease recurrence using a ROC. The recurrence-free survival of the patient group with two or more metastatic LNs was significantly decreased. (The graph was made by statistical software SPSS version 25.0 for windows).

\begin{tabular}{|l|l|l|l|}
\hline Factor & Hazard ratio & $\mathbf{9 5 \%}$ CI & $\boldsymbol{p}$ value \\
\hline Sex & 2.393 & $1.128-5.077$ & 0.023 \\
\hline Size & 1.030 & $1.007-1.052$ & 0.010 \\
\hline ETE & 3.105 & $0.697-13.834$ & 0.137 \\
\hline pT & 1.547 & $0.810-2.954$ & 0.186 \\
\hline pN & 0.790 & $0.170-3.683$ & 0.765 \\
\hline Number of metastatic CLNs & 1.619 & $0.582-4.502$ & 0.356 \\
\hline LNR & 1.337 & $0.480-3.725$ & 0.578 \\
\hline
\end{tabular}

Table 2. Logistic regression analysis for disease recurrence adjusted for clinico-pathologic factors. $C I$ confidence interval, ETE extrathyroidal extension, $L N$ lymph node, $L N R$ lymph node ratio. 


\begin{tabular}{|l|l|l|l|l|}
\hline Model & Accuracy & Precision & Recall & F1 score \\
\hline Decision tree & 0.95 & 0.66 & 0.18 & 0.28 \\
\hline Random forest & 0.91 & 0.25 & 0.27 & 0.26 \\
\hline XGBoost & 0.92 & 0.25 & 0.18 & 0.21 \\
\hline LightGBM & 0.93 & 0.28 & 0.18 & 0.22 \\
\hline Stacking & 0.93 & 0.33 & 0.18 & 0.23 \\
\hline
\end{tabular}

Table 3. Performance results for the machine learning models.

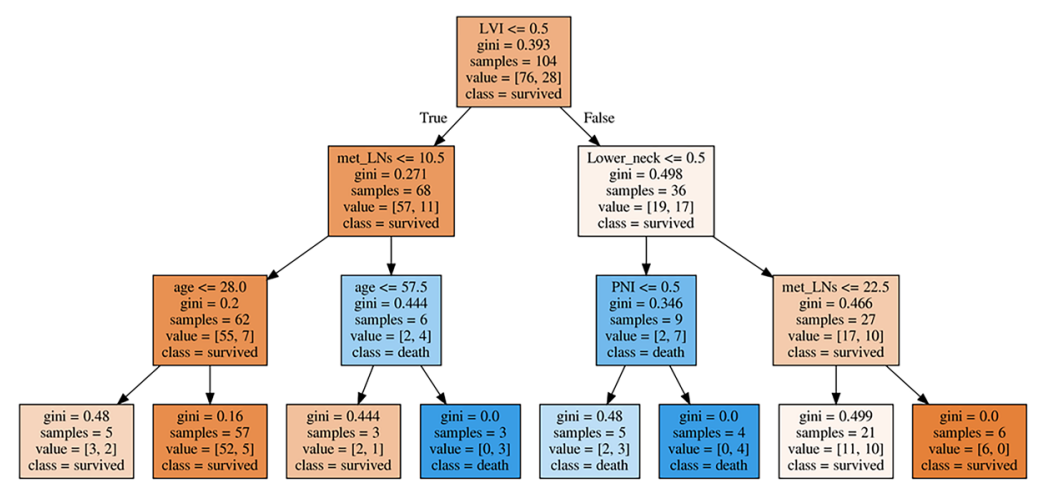

A

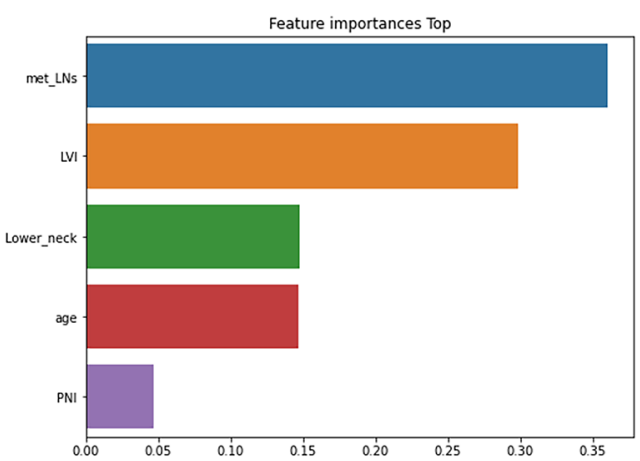

B

Figure 2. The structure of the decision tree model was visualized using graphic software. (The graph was made using by python version 3.8, scikit-learn version 12.3, and graphviz software, All these software are open-sourec program).

\begin{tabular}{|l|l|l|l|l|}
\hline Rank & Decision tree & Random forest & XGBoost & LightGBM \\
\hline 1st & Contralateral CLN metastasis & LNR & Tumor size & Age \\
\hline 2nd & LNR & Ipsilateral CLNs metastasis & Age & Tumor size \\
\hline 3rd & Age & Contralateral CLNs metastasis & Ipsilateral CLNs metastasis & Ipsilateral CLNs metastasis \\
\hline 4th & & Tumor size & LNR & LNR \\
\hline 5th & & Age & Contralateral CLNs metastasis & Contralateral CLNs metastasis \\
\hline
\end{tabular}

Table 4. Top five features of importance among machine learning models. $L N$ lymph node, $L N R$ lymph node ratio.

models do not assume a linear combination of variables used, the effect of correlation between variables can be diminished. Therefore, we can put all those prognostic factors including nodal factors such as LNR and number of metastatic LNs into the model and constructed machine learning prediction model. When analyzing the feature importance of parameters used for machine learning model construction, we noted that contralateral LN metastasis and LNR were used at high frequencies for machine learning model construction in all machine learning models, along with other clinical factors, including tumor size and age.

Among the machine learning techniques used in this study, the decision tree model showed the highest accuracy, followed by the Ensemble models, including LightGBM and stacking techniques. All of the machine learning models showed accuracies of $90 \%$ or more. However, since the models were trained based on data from 1000 patients, more patient data would be required to increase the performance of our models and to apply them in clinical practice. Also, additional research is needed to address the following limitations: Since this was a retrospective study conducted at a single institution, the influence of selection bias cannot be excluded. In addition, considering the indolent features of PTC, a short follow-up period is less optimal for detecting recurrence in PTC patients. Nevertheless, this study is of value as the first study on machine learning models of predicting PTC disease recurrence based clinico-pathologic factors.

In this study, various machine learning models were constructed for predicting disease recurrence in PTC patients, and all of the models had a confirmed accuracy of $90 \%$ or more. In the future, large-scale clinical studies on many patients should be performed to improve the performance of our prediction models, and multicenter clinical studies will be needed to verify their clinical effectiveness. 
Received: 21 November 2020; Accepted: 15 February 2021

Published online: 02 March 2021

\section{References}

1. Altekruse, S., Das, A., Cho, H., Petkov, V. \& Yu, M. Do US thyroid cancer incidence rates increase with socioeconomic status among people with health insurance? An observational study using SEER population-based data. BMJ Open 5(12), e009843 (2015).

2. Grant, C. S. Recurrence of papillary thyroid cancer after optimized surgery. Gland Surg. 4(1), 52-62 (2015).

3. Liu, F. H., Kuo, S. F., Hsueh, C., Chao, T. C. \& Lin, J. D. Postoperative recurrence of papillary thyroid carcinoma with lymph node metastasis. J. Surg. Oncol. 112(2), 149-154 (2015).

4. Lan, X. et al. A Meta-analysis of central lymph node metastasis for predicting lateral involvement in papillary thyroid carcinoma. Otolaryngol. Head Neck Surg. 153(5), 731-738 (2015).

5. Yan, H. et al. A study on central lymph node metastasis in $543 \mathrm{cN} 0$ papillary thyroid carcinoma patients. Int. J. Endocrinol. 2016, 1878194 (2016).

6. Chéreau, N. et al. Recurrence of papillary thyroid carcinoma with lateral cervical node metastases: Predictive factors and operative management. Surgery 159(3), 755-762 (2016).

7. Park, C. H. et al. Significance of the extracapsular spread of metastatic lymph nodes in papillary thyroid carcinoma. Clin. Exp. Otorhinolaryngol. 8(3), 289-294 (2015).

8. Ji, Y. B. et al. Postoperative hypoparathyroidism and the viability of the parathyroid glands during thyroidectomy. Clin. Exp. Otorhinolaryngol. 10(3), 265-271 (2017).

9. Podnos, Y. D., Smith, D., Wagman, L. D. \& Ellenhorn, J. D. The implication of lymph node metastasis on survival in patients with well-differentiated thyroid cancer. Am. Surg. 71(9), 731-734 (2005).

10. Zaydfudim, V., Feurer, I. D., Griffin, M. R. \& Phay, J. E. The impact of lymph node involvement on survival in patients with papillary and follicular thyroid carcinoma. Surgery. 144(6), 1070-1071 (2008).

11. Sugitani, I., Kasai, N., Fujimoto, Y. \& Yanagisawa, A. A novel classification system for patients with PTC: Addition of the new variables of large (3 cm or greater) nodal metastases and reclassification during the follow-up period. Surgery 135(2), 139-148 (2004).

12. Park, Y. M. et al. Metastatic lymph node status in the central compartment of papillary thyroid carcinoma: A prognostic factor of locoregional recurrence. Head Neck. 38(Suppl 1), E1172-E1176 (2016).

13. Vas Nunes, J. H. et al. Prognostic implications of lymph node yield and lymph node ratio in papillary thyroid carcinoma. Thyroid 23(7), 811-816 (2013).

14. Momesso, D. P. \& Tuttle, R. M. Update on differentiated thyroid cancer staging. Endocrinol. Metab. Clin. N. Am. 43(2), 401-421 (2014).

15. Haugen, B. R. 2015 American thyroid association management guidelines for adult patients with thyroid nodules and differentiated thyroid cancer: What is new and what has changed?. Cancer 123(3), 372-381 (2017).

16. Kim, T. H. et al. Prognostic value of the eighth edition AJCC TNM classification for differentiated thyroid carcinoma. Oral Oncol. 71, 81-86 (2017)

17. Baek, S. K. et al. Clinical risk factors associated with cervical lymph node recurrence in papillary thyroid carcinoma. Thyroid. 20(2), 147-152 (2010)

18. Lang, B. H., Chow, S. M., Lo, C. Y., Law, S. C. \& Lam, K. Y. Staging systems for papillary thyroid carcinoma: a study of 2 tertiary referral centers. Ann. Surg. 246(1), 114-121 (2007).

19. Lupi, C. et al. Association of BRAF V600E mutation with poor clinicopathological outcomes in 500 consecutive cases of papillary thyroid carcinoma. J. Clin. Endocrinol. Metab. 92(11), 4085-4090 (2007).

20. Xing, M. et al. Association between BRAF V600E mutation and mortality in patients with papillary thyroid cancer. JAMA 309(14), 1493-1501 (2013).

21. Randolph, G. W. et al. American Thyroid Association Surgical Affairs Committee's Taskforce on Thyroid Cancer Nodal Surgery. The prognostic significance of nodal metastases from papillary thyroid carcinoma can be stratified based on the size and number of metastatic lymph nodes, as well as the presence of extranodal extension. Thyroid 22(11), 1144-1152 (2012).

22. Haugen, B. R. et al. 2015 American thyroid association management guidelines for adult patients with thyroid nodules and differentiated thyroid cancer: The American thyroid association guidelines task force on thyroid nodules and differentiated thyroid cancer. Thyroid 26(1), 1-133 (2016).

23. Mazo, C., Bernal, J., Trujillo, M. \& Alegre, E. Transfer learning for classification of cardiovascular tissues in histological images. Comput. Methods Prog. Biomed. 165, 69-76 (2018).

24. Karri, S. P., Chakraborty, D. \& Chatterjee, J. Transfer learning based classification of optical coherence tomography images with diabetic macular edema and dry age-related macular degeneration. Biomed. Opt. Express 8(2), 579-592 (2017).

25. Gulshan, V. et al. Development and validation of a deep learning algorithm for detection of diabetic retinopathy in retinal fundus photographs. JAMA 316(22), 2402-2410 (2016).

26. Kermany, D. S. et al. Identifying medical diagnoses and treatable diseases by image-based deep learning. Cell 172(5), 1122-1131 (2018).

27. Hood, D. C. \& De Moraes, C. G. Efficacy of a deep learning system for detecting glaucomatous optic neuropathy based on color fundus photographs. Ophthalmology 125(8), 1207-1208 (2018).

28. Tschandl, P. et al. Comparison of the accuracy of human readers versus machine-learning algorithms for pigmented skin lesion classification: an open, web-based, international, diagnostic study. Lancet Oncol. 20(7), 938-947 (2019).

29. Ito, Y. et al. Prognosis of patients with papillary thyroid carcinoma showing postoperative recurrence to the central neck. World J. Surg. 35(4), 767-772 (2011).

30. Tuttle, R. M., Haugen, B. \& Perrier, N. D. Updated American joint committee on cancer/tumor-node-metastasis staging system for differentiated and anaplastic thyroid cancer (eighth edition): What changed and why?. Thyroid 27(6), 751-756 (2017).

31. Nixon, I. J. et al. Defining a valid age cutoff in staging of well-differentiated thyroid cancer. Ann. Surg. Oncol. 23(2), 410-415 (2016).

32. Schneider, D. F., Chen, H. \& Sippel, R. S. Impact of lymph node ratio on survival in papillary thyroid cancer. Ann. Surg. Oncol. 20(6), 1906-1911 (2013)

33. Ryu, I. S. et al. Lymph node ratio of the central compartment is a significant predictor for locoregional recurrence after prophylactic central neck dissection in patients with thyroid papillary carcinoma. Ann. Surg. Oncol. 21(1), 277-283 (2014).

34. Lee, J. et al. Clinical value of lymph node ratio integration with the 8(th) edition of the UICC TNM classification and 2015 ATA risk stratification systems for recurrence prediction in papillary thyroid cancer. Sci. Rep. 9(1), 13361 (2019).

\section{Acknowledgements}

This study was supported by a Research Grant from Gangnam Severance Hospital, Yonsei University College of Medicine. 


\section{Author contributions}

Y.M.P designed the study and collected data. Y.M.P wrote the machine learning code and performed training of it. Y.M.P drafted the manuscript with B.Y.L.

\section{Competing interests}

The authors declare no competing interests.

\section{Additional information}

Correspondence and requests for materials should be addressed to Y.M.P. or B.-J.L.

Reprints and permissions information is available at www.nature.com/reprints.

Publisher's note Springer Nature remains neutral with regard to jurisdictional claims in published maps and institutional affiliations.

(c) (i) Open Access This article is licensed under a Creative Commons Attribution 4.0 International License, which permits use, sharing, adaptation, distribution and reproduction in any medium or format, as long as you give appropriate credit to the original author(s) and the source, provide a link to the Creative Commons licence, and indicate if changes were made. The images or other third party material in this article are included in the article's Creative Commons licence, unless indicated otherwise in a credit line to the material. If material is not included in the article's Creative Commons licence and your intended use is not permitted by statutory regulation or exceeds the permitted use, you will need to obtain permission directly from the copyright holder. To view a copy of this licence, visit http://creativecommons.org/licenses/by/4.0/.

(C) The Author(s) 2021 